\title{
Interaction of molecular and atomic hydrogen with $(5,5)$ and $(6,6)$ single-wall carbon nanotubes
}

\author{
J. S. Arellano \\ Area de Física Atómica Molecular Aplicada, Universidad Autónoma Metropolitana Azcapotzalco, \\ C.P. 02200, México D.F., México \\ L. M. Molina \\ Institute of Physics and Astronomy, University of Aarhus, DK-8000 Aarhus C, Denmark \\ A. Rubio \\ Departamento de Física de Materiales, Universidad del País Vasco, 20018 San Sebastián, Spain \\ M. J. López and J. A. Alonso \\ Departamento de Física Teórica, Universidad de Valladolid, E-47011, Valladolid, Spain
}

(Received 12 March 2002; accepted 2 May 2002)

\begin{abstract}
Density functional theory has been used to study the interaction of molecular and atomic hydrogen with $(5,5)$ and $(6,6)$ single-wall carbon nanotubes. Static calculations allowing for different degrees of structural relaxation are performed, in addition to dynamical simulations. Molecular physisorption inside and outside the nanotube walls is predicted to be the most stable state of those systems. The binding energies for physisorption of the $\mathrm{H}_{2}$ molecule outside the nanotube are in the range $0.04-0.07 \mathrm{eV}$. This means that uptake and release of molecular hydrogen from nanotubes is a relatively easy process, as many experiments have proved. A chemisorption state, with the molecule dissociated and the two hydrogen atoms bonded to neighbor carbon atoms, has also been found. However, reaching this dissociative chemisorption state for an incoming molecule, or starting from the physisorbed molecule, is difficult because of the existence of a substantial activation barrier. The dissociative chemisorption deforms the tube and weakens the $\mathrm{C}-\mathrm{C}$ bond. This effect can catalyze the shattering and scission of the tube by incoming hydrogen molecules with sufficient kinetic energy. (c) 2002 American Institute of Physics. [DOI: 10.1063/1.1488595]
\end{abstract}

\section{INTRODUCTION}

Motivated by the search for materials with good capacity for storing hydrogen, the absorption of hydrogen by carbon nanotubes and graphitic fibers has been investigated by several groups. ${ }^{1-14}$ The results are controversial, with reports of high absorption capacity alternating with less optimistic ones. Additional experiments on well-controlled samples are needed to resolve the discrepancies between experimentalists. Also, theoretical calculations may be very useful to understand the nature of the absorption and storage processes. ${ }^{15}$ Computer simulations of the hydrogen adsorption have been performed using model potentials to describe the interactions. ${ }^{7,16-19}$ The use of empirical potentials is convenient for massive simulations, but only $a b$ initio calculations can give an accurate description of the interaction of hydrogen with the graphitic fibers.

Using density functional theory (DFT), Jeloaica and Sidis $^{20}$ calculated the interaction between atomic hydrogen and a graphite surface. Similar calculations have been performed to study the interaction of atomic hydrogen with single-wall nanotubes (SWNT's), ${ }^{21-24}$ and Gülseren and co-workers ${ }^{24}$ found that the binding energy depends sensitively on the curvature of the nanotube. Lee and Lee ${ }^{23}$ performed DFT and tight-binding calculations to search for $\mathrm{H}$ chemisorption sites in SWNT's. Although the chemisorption of atomic hydrogen is an important issue, the technologically relevant process is the physisorption of molecular hydrogen on the walls outside or inside the nanotubes. Using DFT we have investigated the adsorption of $\mathrm{H}_{2}$ on a planar graphene layer, ${ }^{25}$ and the interaction between $\mathrm{H}_{2}$ and SWNT nanotubes has been studied in some recent papers. ${ }^{26-29}$ Tada et al. ${ }^{26}$ reported a purely repulsive interaction between $\mathrm{H}_{2}$ and a planar graphene layer, a prediction in disagreement with our previous result ${ }^{25}$ and with the experiments for adsorption on graphite pores, nanotube bundles, and nanofibers. The discrepancy between the results of the two calculations is due to the different treatments of exchange and correlation effects: the local density approximation (LDA) was used by Arellano et al. ${ }^{25}$ and the generalized gradient approximation (GGA) by Tada et $_{\text {al. }}{ }^{26}$ The GGA has been criticized $^{30}$ for giving a purely repulsive interaction between systems characterized by weak bonding forces, as occurs for the $\mathrm{H}_{2}$-graphite system. A recent study of the interaction of molecular hydrogen with planar graphitic clusters by Okamoto and Miyamoto ${ }^{29}$ has confirmed that the LDA predicts physisorption of the molecule, while the GGA and hybrid-DFT methods lead to a repulsive interaction. Furthermore, the LDA interaction energy curve was in very good agreement with the results of second-order Møller-Plesset (MP2) calculations performed by the same authors.

Tada et $a l^{26}$ also find a repulsive interaction between $\mathrm{H}_{2}$ and $(6,6)$ carbon nanotubes; however, at short distances from the nanotube wall the molecule undergoes dissociative 
chemisorption, but a barrier of $2 \mathrm{eV}$ has to be overcome. The magnitude of this barrier becomes reduced for tubes of very small diameter: $(4,4)$ and $(3,3)$ nanotubes. The calculations of Chan and co-workers ${ }^{28}$ also give a purely repulsive interaction between $\mathrm{H}_{2}$ and $(6,6)$ nanotubes at ordinary pressure, but under high external pressure they predict dissociative chemisorption on $(6,6)$ SWNT bundles.

In our opinion the interplay between molecular physisorption and atomic chemisorption is still an open question and in this paper we use DFT to investigate the interaction of atomic and molecular hydrogen with $(5,5)$ and $(6,6)$ nanotubes, both outside and inside the tubes. For this purpose we perform static calculations as well as dynamical simulations of the process of an $\mathrm{H}_{2}$ molecule approaching a nanotube. The main purpose of our work is to compare the possibilities for molecular physisorption and dissociative atomic chemisorption.

\section{COMPUTATIONAL METHOD}

The calculations of the interaction between the hydrogen atom, or the hydrogen molecule, and the carbon nanotubes have been performed with the ab initio FHI96MD code. ${ }^{31}$ This code uses the DFT to compute the electron density and the total energy of the system, and we have employed the LDA to account for exchange and correlation effects. ${ }^{32}$ The code uses a triangular supercell geometry and a plane-wave expansion for the electronic wave functions. Only the electrons of the hydrogen atoms and the external electrons $\left(2 s^{2} 2 p^{2}\right)$ of the carbon atoms are explicitly considered in the calculations, while the $1 s^{2}$ core of each carbon atom is replaced by a pseudopotential. For this purpose we have selected the nonlocal norm-conserving pseudopotentials of Hamman and co-workers. ${ }^{33}$ Nonlocality in the carbon pseudopotential is restricted to $l=2$, and we take the $s$ component as the local part. A kinetic energy cutoff of $40 \mathrm{Ry}$ for the plane-wave expansion of the electronic wave functions was used in most calculations. This is similar to the cutoff chosen by Tada et $a .^{26}$ (44 Ry), but in selected cases, to be indicated in the text, we have used higher cutoff energies. A single $(\Gamma)$ point was used in the Brillouin zone sampling.

Below we present results for static calculations and dynamical simulations for the interaction of $\mathrm{H}$ and $\mathrm{H}_{2}$ with $(5,5)$ and $(6,6)$ nanotubes. The supercell, which contains 20 carbon atoms in the case of $(5,5)$ nanotubes, is defined by the lattice parameters $a=28.96$ a.u. and $c=4.64$ a.u. In selected cases, to be explicitly indicated in the text, we have used a larger value for $a$. In the triangular supercell lattice, the distance between the axes of two adjacent nanotubes is given by the cell parameter $a$. A bigger supercell with 48 carbon atoms is used in the case of $(6,6)$ nanotubes. In that case, the value of $a$ is again $a=28.96$ a.u., but the value of $c$ has been doubled, $c=9.28$ a.u. In addition, several dynamical simulations were done for the $(6,6)$ nanotube system, with the same $c$ parameter as for the $(5,5)$ nanotube and 24 carbon atoms.

The presentation of our results is structured in a way that allows to appreciate the effect of the relaxation of interatomic distances. First, static calculations are performed in which the $\mathrm{H}_{2}$ bond length, as well as the $\mathrm{C}-\mathrm{C}$ distances in the nanotube, is frozen and only the molecule-SWNT dis-

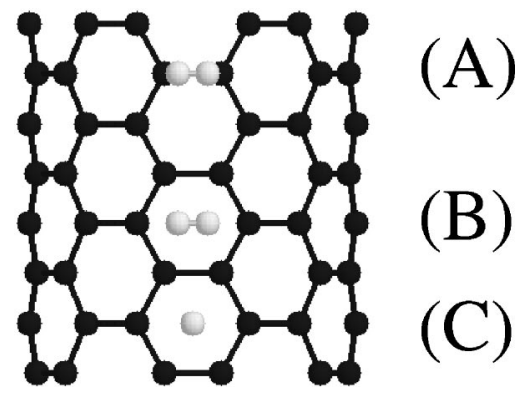

FIG. 1. Physisorption configurations with the axis of the $\mathrm{H}_{2}$ molecule above a $\mathrm{C}-\mathrm{C}$ bond $(\mathrm{A})$ and above a hexagon with the molecular axis parallel (B) or perpendicular $(\mathrm{C})$ to the hexagon surface.

tance, or the H-SWNT distance, is allowed to vary. Those calculations have the virtue of allowing us to plot the potential energy of the system in terms of a single variable: the distance between the center of mass of the $\mathrm{H}_{2}$ molecule and the nanotube wall. Then, another set of static calculations is performed in which the $\mathrm{H}-\mathrm{H}$ distance is allowed to vary while maintaining all the $\mathrm{C}-\mathrm{C}$ distances in the SWNT still frozen. In a third stage, damped dynamical simulations are performed in which the molecule is first placed at a certain distance from the SWNT and the whole system is then allowed to dynamically relax by the action of the instantaneous forces on all the atoms, until it reaches a nearby energy minimum in the potential energy surface. In those simulations, the initial $\mathrm{H}-\mathrm{H}$ bond length is sometimes that of the free molecule, but we have also started with configurations corresponding to a dissociated molecule.

\section{INTERACTION OF $\mathrm{H}_{2}$ AND H WITH $(5,5)$ CARBON NANOTUBES: STATIC CALCULATIONS}

The radial approach of the $\mathrm{H}_{2}$ molecule to the SWNT surface following a pathway with the molecular axis parallel to a bond between two nearest-neighbor carbon atoms on the nanotube was considered by Tada et $_{\text {al. }}{ }^{26}$ as the most probable pathway for dissociative adsorption. Then, after an initial optimization of the structure of the free $(5,5)$ SWNT, which gives an average $\mathrm{C}-\mathrm{C}$ bond length of 2.68 a.u. and a nanotube radius $R=6.44$ a.u., a hydrogen molecule was placed upon the middle point of a $\mathrm{C}-\mathrm{C}$ bond and parallel to that bond (configuration A in Fig. 1), and the energy of the system was calculated as a function of the distance $D_{a}$ from the center of mass of the molecule to the nanotube axis. In these initial calculations the distance between the two $\mathrm{H}$ atoms was mantained fixed, equal to 1.48 a.u., the equilibrium bond length of the free molecule obtained previously, ${ }^{25}$ and the coordinates of all the carbon atoms of the SWNT were also kept frozen. Neglecting the relaxation of the interatomic distances imposes rather restrictive conditions, but allows us to plot the interaction energy curve in terms of a single parameter, the distance $D_{a}$, and this curve can serve as a reference for the more realistic calculations including relaxation to be presented below. Weak physisorption wells are obtained for the molecule inside and outside the nanotube, and the absolute minimum of the potential energy occurs for the molecule at the center of the tube. The interaction energy 




FIG. 2. Interaction energy of the $\mathrm{H}_{2}$ molecule and a $(5,5) \mathrm{SWCN}$, obtained in static calculations in which the $\mathrm{H}-\mathrm{H}$ and all the $\mathrm{C}-\mathrm{C}$ bond lengths are kept frozen, and only the distance $D_{a}$ between the molecule and the SWNT axis is varied. In the approach of the molecule to the nanotube, the center of mass of the molecule is upon the midpoint of a $\mathrm{C}-\mathrm{C}$ bond with the molecular axis parallel to that bond (circles) or upon the center of a hexagon with the molecular axis parallel (crosses) or perpendicular (diamonds) to the hexagon surface.

curve is given in Fig. 2. This is the curve joining the circles. We have subtracted the asymptotic value of the energy obtained for a sufficiently large distance $D_{a}$. The external minimum occurs for $D_{a}$ near 12.0 a.u., that is, at a distance $D_{w}$ about 5.5 a.u. between the molecule and the nanotube wall $\left(D_{w}=D_{a}-R\right)$, and its depth is $0.043 \mathrm{eV}$. The interaction rapidly becomes highly repulsive as the molecule approaches the nanotube wall. The attractive well is deeper for the molecule inside the tube (its depth is $0.17 \mathrm{eV}$ ), an effect of the opposite curvature inside and outside the nanotube. Inside the nanotube the molecule effectively sees a larger nanotube area, and the energy minimum coincides with the nanotube axis because the nanotube radius is only slightly larger than the optimun physisorption distance. The enhancement factor for the binding energy, $E_{\mathrm{b}}$ (inside) $/ E_{\mathrm{b}}$ (outside), is consistent with the MP2 results obtained by Okamoto and Miyamoto $^{29}$ for planar and curved graphene clusters.

Some tests were performed to investigate the sensitivity of the results to details of the computational method. Enlarging the lattice parameter $a$ from 28.96 a.u. to 40 a.u. has a negligible effect on the binding energy of the molecule: the binding energy $E_{\mathrm{b}}$ outside the nanotube is again $0.043 \mathrm{eV}$. The same value for the binding energy and a nearly identical position of the minimum were also obtained when the lattice parameter is 28.96 a.u. and the plane-wave cutoff energy is increased from the previous value of 40 Ry to $70 \mathrm{Ry}$.

The results for the radial approach of the molecule upon the center of a hexagon of carbon atoms, with the molecular axis parallel and perpendicular to the hexagon surface (configurations $\mathrm{B}$ and $\mathrm{C}$ in Fig. 1), are also shown in Fig. 2. The inner minimum lies again at the nanotube center in both cases. The outer one occurs at $D_{a}=11.5$ a.u. and $D_{a}$ $=11.4$ a.u. for the perpendicular and parallel configurations, respectively, that is, at distances $D_{w}$ of 5.1 a.u. and 5.0 a.u. from the nanotube wall. The binding energies outside the nanotube are the same, $E_{\mathrm{b}}=0.068 \mathrm{eV}$, consistent with the experimental value of $0.062 \mathrm{eV}$ obtained by Brown et al. ${ }^{34}$ by thermally activated $\mathrm{H}_{2}$ desorption from nanotubes with radius about 13 a.u. The calculated position and depth of the physisorption well are consistent with the corresponding values obtained for the interaction of $\mathrm{H}_{2}$ with a planar graphene layer $^{25}$ : the equilibrium distance of the molecule to the planar graphene layer in the parallel configuration upon a hexagon (the most stable configuration) is 5.07 a.u. and the binding energy is $0.086 \mathrm{eV}$. The slightly lower binding to the nanotube is a curvature effect, but in both cases, graphene and nanotube, the position of the minimum of the outer physisorption well occurs for the molecule above the center of a carbon hexagon. The depth of the physisorption well for the graphene layer is in perfect agreement with the result of an MP2 calculation, ${ }^{29}$ which also gives a binding energy of $0.086 \mathrm{eV}$. These binding energies are also consistent with the values used in the empirical potentials, ${ }^{7,16}$ which range between 0.03 and $0.05 \mathrm{eV}$.

Although it is known that the LDA does not account for dispersion forces between molecules, which strictly act in the limit of negligible overlap between the electron densities, it usually gives reasonable results for the interactions in the region of small overlap for systems with weak interaction forces, that is, in the region of the physisorption minimum. ${ }^{30,35-38}$ The main reason is the nonlinearity of the exchange energy density functional

$$
e_{x}(\mathbf{r})=-\frac{3}{4}(3 / \pi)^{1 / 3} n(\mathbf{r})^{4 / 3}
$$

which means that even without density redistribution, the exchange energy density of the interacting system in the region of space where the densities overlap, namely, $e_{x}(\mathbf{r})=-\frac{3}{4}(3 / \pi)^{1 / 3}\left[n_{1}(\mathbf{r})+n_{2}(\mathbf{r})\right]^{4 / 3}$, is more negative than the sum of the corresponding energy densities of the individual molecules. The presence of a physisorption well is consistent with the abundant experimental information on absorption of $\mathrm{H}_{2}$ by SWNT's and graphitic fibers.

It is natural to expect that the preferred site for the absorption of a single hydrogen atom on a SWNT is on top of a carbon atom ${ }^{20-24}$; in fact, the $\mathrm{C}-\mathrm{H}$ stretching mode has been observed using Fourier transform infrared spectroscopy. ${ }^{34}$ So we have studied the radial approach of the hydrogen atom to the nanotube following a path on top of a $\mathrm{C}$ atom. The interaction energy has a minimum for $D_{a}$ near 9.0 a.u. (2.6 a.u. from the nanotube wall), and the binding energy is $0.9 \mathrm{eV}$. Also we calculated the energy of interaction for the perpendicular approach of the hydrogen atom upon the midpoint of a carbon-carbon bond. The corresponding interaction energy curve is less attractive, with a binding energy of $0.46 \mathrm{eV}$, but at the same time it is less repulsive at short distances from the wall. The lower binding energy suggests that the configuration corresponding to the minimum of the latter curve is simply the saddle point for the migration of the $\mathrm{H}$ atom from its equilibrium position upon a $\mathrm{C}$ atom to another equivalent equilibrium position upon an adjacent $\mathrm{C}$ atom following a path along the $\mathrm{C}-\mathrm{C}$ bond. The migration energy barrier is $0.45 \mathrm{eV}$. The two curves just described have been plotted in Fig. 3. These are the curve joining the triangles and the curve joining the squares, respectively, al- 


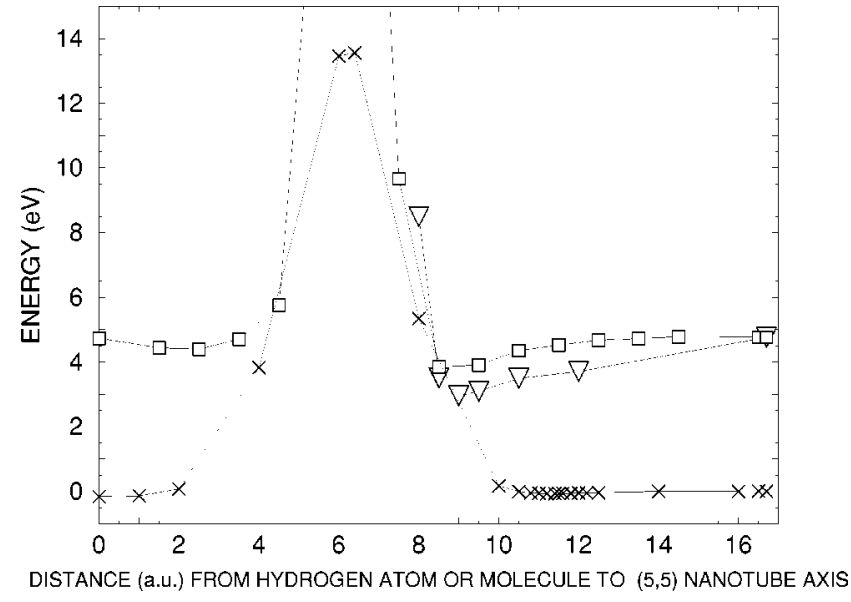

FIG. 3. Interaction energies for the radial approach of atomic and molecular hydrogen to a $(5,5) \mathrm{SWCN}$, obtained in static calculations similar to those reported in Fig. 2. The approach of the $\mathrm{H}_{2}$ molecule is upon the center of a hexagon, with the molecular axis parallel to the hexagon surface (crosses). The approach of the hydrogen atom is upon a carbon atom (triangles) or upon the midpoint of a $\mathrm{C}-\mathrm{C}$ bond (squares). The energies of the curves for atomic $\mathrm{H}$ have been multiplied by a factor of 2 and shifted upwards (see text).

though each one of those potential energy curves has been multiplied by a factor of 2 , for reasons to be discussed below.

The binding energies of a $\mathrm{H}$ atom to graphitic surfaces (nanotubes and graphene) calculated using DFT show a wide range of values, and our results lie within that range. Gülseren et al. ${ }^{24}$ obtained binding energies to nanotubes higher than $2 \mathrm{eV}$. The extrapolation of their results to a nanotube of infinite radius (planar graphene) gives $E_{\mathrm{b}}=1.49 \mathrm{eV}$. Bauschlicher ${ }^{21}$ obtained $E_{\mathrm{b}}=0.93 \mathrm{eV}$ for a $(10,10)$ nanotube. On the other hand, Jeloaica and Sidis ${ }^{20}$ have calculated the binding energy of the $\mathrm{H}$ atom to a graphene layer simulated by a finite cluster using different exchange-correlation (xc) functionals. The binding energies spanned a range of values between 0.1 and $0.6 \mathrm{eV}$, depending on the xc functional and the degree of geometrical relaxation allowed for the $\mathrm{C}$ atoms on the cluster, with the upper value of $0.6 \mathrm{eV}$ corresponding to the best calculation. In summary, considering the spread of values obtained in previous calculations for nanotubes and graphene, we consider that the value near $1 \mathrm{eV}$ that we have obtained for the binding energy of the $\mathrm{H}$ atom to the $(5,5)$ nanotube is a reasonable result.

The curves giving the interaction of the hydrogen atom and the nanotube plotted in Fig. 3 have been multiplied by a factor of 2 in order to compare those curves with the interaction of a $\mathrm{H}_{2}$ molecule with the same nanotube. The interaction potential between $\mathrm{H}_{2}$ and the nanotube, corresponding to the case of the molecule parallel upon a hexagon, has also been included in Fig. 3 (notice that the energy scales of Figs. 2 and 3 are very different). Furthermore, to compare molecular and atomic absorption, the curves representing atomic absorption have been displaced with respect to the molecular absorption curve by a constant shift of $4.76 \mathrm{eV}$, which is the experimental dissociation energy of the hydrogen molecule in two $\mathrm{H}$ atoms (the value that we calculate using the spinpolarized LDA is very close to this quantity). The fact that the curve for the $\mathrm{H}$ atom on top of a $\mathrm{C}$ atom has a minimum

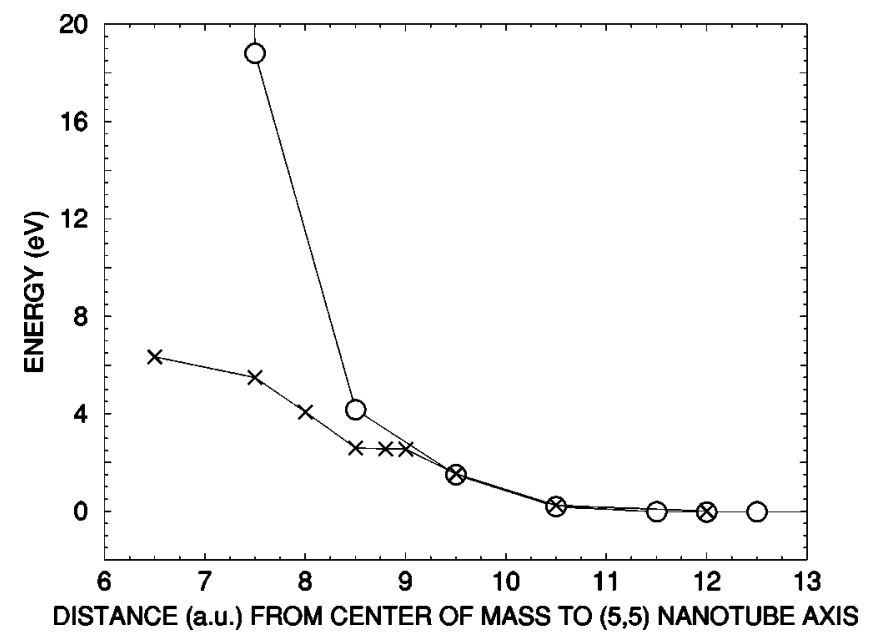

FIG. 4. Interaction energies between $\mathrm{H}_{2}$ and a $(5,5)$ nanotube. The molecule is upon a $\mathrm{C}-\mathrm{C}$ bond. The $\mathrm{C}-\mathrm{C}$ distances are kept frozen. The $\mathrm{H}-\mathrm{H}$ distance is maintained frozen in the upper curve (circles), and it is optimized in the lower curve (crosses).

indicates that two (or more) $\mathrm{H}$ atoms can be chemisorbed on the surface of the nanotube, provided that those two $\mathrm{H}$ atoms are sufficiently separated from each other. However, this is not the most stable state of the system. The prediction is that in the most stable state the two $\mathrm{H}$ atoms form a bound molecule, physisorbed on the surface of the nanotube. A lower bound for the energy barrier for the dissociation of the $\mathrm{H}_{2}$ molecule on the nanotube surface can be obtained by subtracting the energies of the minima of the atomic and molecular curves in Fig. 3. This rough estimation gives $3 \mathrm{eV}$. The prediction of molecular physisorption is also valid on the inner wall of the nanotube.

\section{INTERACTION OF $\mathrm{H}_{2}$ AND H WITH $(5,5)$ CARBON NANOTUBES: CALCULATIONS INCLUDING ATOMIC RELAXATION}

Next we have performed calculations in which, for each separation $D_{a}$ between the molecule and the nanotube axis, the distance $d$ between the two hydrogen atoms is allowed to vary to minimize the energy of the system, still maintaining frozen the positions of the carbon atoms of the nanotube; this was done by performing several static calculations corresponding to different values of $d$. The orientation of the molecule was parallel upon a $\mathrm{C}-\mathrm{C}$ bond. For distances $D_{a}$ larger than 9.5 a.u. there is practically no relaxation of the bond length of the molecule. However, for closer approach to the nanotube, that is, well inside the repulsive part of the $\mathrm{H}_{2}$-SWNT interaction potential of Fig. 2, there is an appreciable $\mathrm{H}-\mathrm{H}$ bond length relaxation. For instance, when $D_{a}=8.5$ a.u., the $\mathrm{H}-\mathrm{H}$ distance $d$ increases up to 3.3 a.u.; that is, the molecule dissociates (notice that the bond length of the free molecule is 1.48 a.u.), and there is an associated lowering of the energy of the system. For even shorter $D_{a}$ separations, the two $\mathrm{H}$ atoms are pulled farther apart from each other. That is, relaxation of the molecular bond length lowers the energy of the system for values of $D_{a}$ smaller than 9.5 a.u., as we show in Fig. 4 by a comparison of the $\mathrm{H}_{2}$-nanotube potential energy curves without (circles) and 
with (crosses) relaxation of the molecular bond length (we stress that the $\mathrm{H}-\mathrm{H}$ distance is not constant for the later curve). A shallow plateau now appears in the region $D_{a}$ $=8.5-9.0$ a.u. $\left(D_{w}=2.1-2.6\right.$ a.u. $)$ of the later curve. The $\mathrm{H}-\mathrm{H}$ distances in that region are in the range $d$ $=3.1-3.3$ a.u. This feature, combined with the analysis of Fig. 3, indicates that the molecule dissociates when forced to approach sufficiently close to the nanotube wall. However, relaxation of the molecular bond length does not alter the main picture, and the absolute energy minimum of the system occurs for the molecule physisorbed at a distance $D_{a}$ near 12 a.u. The energy barrier required to reach the dissociative chemisorption state, measured from the energy of the physisorption minimum, is $2.6 \mathrm{eV}$, a value consistent with the estimation given in Sec. III above.

To complement the static calculations we have performed damped molecular dynamics simulations, starting from several initial configurations and dynamically relaxing the system under the action of the forces on the atoms. First of all we performed a simulation with initial conditions $D_{a}$ $=9.5$ a.u. and $d=1.47$ a.u. The dynamical evolution of the system drives the molecule towards the physisorption minimum. In the final configuration, $d=1.48$ a.u., and the nanotube shows no distortion. The energy of the system in this configuration is almost identical to that obtained in the static calculation for $D_{a}=12$ a.u. The simulation also shows that the axis of the physisorbed molecule changes easily its orientation with respect to the SWNT axis. To analyze the sensitivity of the simulations to the cell parameter $a$, dynamical simulations were performed with a larger cell parameter $a$ $=40$ a.u. and initial conditions $D_{a}=12.5$ a.u., $d=1.47$ a.u. The simulations produce a minimal increase of $d$ ( $d$ $=1.48$ a.u.) and leave the molecule in the physisorption well, with the $\mathrm{H}$ atoms at equal distance of 12.1 a.u. from the nanotube axis, so the results are consistent with those obtained with the smaller cell parameter ( $a=28.96$ a.u.).

On the other hand, several simulations were performed starting with a dissociated molecule initially placed at separations between its center of mass and the nanotube axis in the range $D_{a}=7.5-8.5$ a.u., that is, at close distances $D_{w}$ $=1.1-2.1$ a.u. from the nanotube wall. The analysis of the sequence of configurations as the simulation develops in time shows that the circular cross section of the nanotube becomes substantially deformed due to the strong attachment of the two hydrogen atoms to a pair of nearest carbon atoms. A snapshot taken from the simulation is given in Fig. 5, where the structure plotted contains two simulations cells, in order to improve visualization of the system. In this configuration the $\mathrm{C}-\mathrm{H}$ bond distances reach values of 2.1 a.u., a bond length shorter than the 2.6 a.u. bond length found for the absorption of a single atom. The distance between those two particular carbon atoms is elongated up to 3.01 a.u. This distance is substantially larger than the $\mathrm{C}-\mathrm{C}$ bond length in the free nanotube (2.68 a.u.). It is even larger than the $\mathrm{C}-\mathrm{C}$ bond length in diamond (2.92 a.u.), the prototypical $s p^{3}$-bonded carbon material. The $\mathrm{C}-\mathrm{C}$ bond is at the verge of breaking and the shortening of the $\mathrm{C}-\mathrm{H}$ bond length seems itself a consequence of the weakening of the $\mathrm{C}-\mathrm{C}$ bond. Our interpretation is that the $\mathrm{C}-\mathrm{C}$ bond is breaking

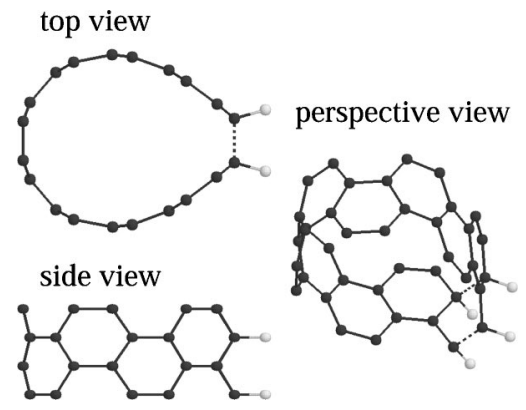

FIG. 5. Configuration snapshot at the verge of nanotube scission for a dynamical simulation starting with a dissociated hydrogen molecule with its center of mass initially at a distance $D_{a}=8.5$ a.u. from the axis of a $(5,5)$ carbon nanotube. Three views are provided. The two hydrogen atoms of the molecule become attached to neighbor carbon atoms (the two $\mathrm{H}-\mathrm{C}$ distances are aproximately 2.14 a.u.), and the nanotube is substantially deformed. The weakened $\mathrm{C}-\mathrm{C}$ bond is breaking and this is indicated by the dashed line between the two $\mathrm{C}$ atoms. To improve the visualization of the system, the structure shown contains two simulation cells along the direction of the nanotube axis.

by the combined effects of (a) the weakening of the $\mathrm{C}-\mathrm{C}$ bond by the concerted chemisorption of the two $\mathrm{H}$ atoms and (b) the excess energy initially deposited on the system, since the simulations begin from configurations of high potential energy. One has to keep in mind that we have performed the simulations for a periodic super lattice. Because of the periodic repetition along the direction of the nanotube axis, there is a breaking $\mathrm{C}-\mathrm{C}$ bond in each unit cell of the nanotube, so the nanotube is cut along a line parallel to the nanotube axis. Beyond this point the scission of the nanotube produces a pronounced instability of the simulation. Scission suddenly opens a new region in the potential energy landscape, where the broken nanotube follows a steep path towards the formation of a planar graphene sheet, but this process cannot be handled by our simulations because the dimensions of the simulation cell (in particular, the value of $a$ ) are not big enough to accomodate the unfolding. We present additional evidence of scission for the case of the $(6,6)$ nanotubes in Sec. VI below. Before going further we stress that a configuration of high potential energy $\left(\mathrm{H}_{2}\right.$ molecules very close to the nanotube wall) is required for the breaking of $\mathrm{C}-\mathrm{C}$ bonds. However, it is unlikely that the $\mathrm{H}_{2}$ molecules will reach those regions of the potential energy surface under normal conditions realized in absorption experiments (see, neverheless, further comments in Sec. VI).

Our simulations have analyzed the effect of a strip of aligned dissociated $\mathrm{H}-\mathrm{H}$ pairs. Attempts to study more complex configurations than the strip would require enlarging the size of the simulation cell. Since the scission effect we have found only occurs under rather special conditions, we have not pursued this point. However, the study of different patterns under normal absorption conditions (no scission) will be worth pursuing and some interesting work has already been done on this topic. Experimental observations of the positions occupied by halogen atoms absorbed outside or inside single wall nanotubes show that those atoms form well organized patterns and not random arrangements. ${ }^{39,40}$ Iodine atoms incorporated inside SWNT's form helical chains ${ }^{39}$ and fluorinated SWNT's reveal a structure where 


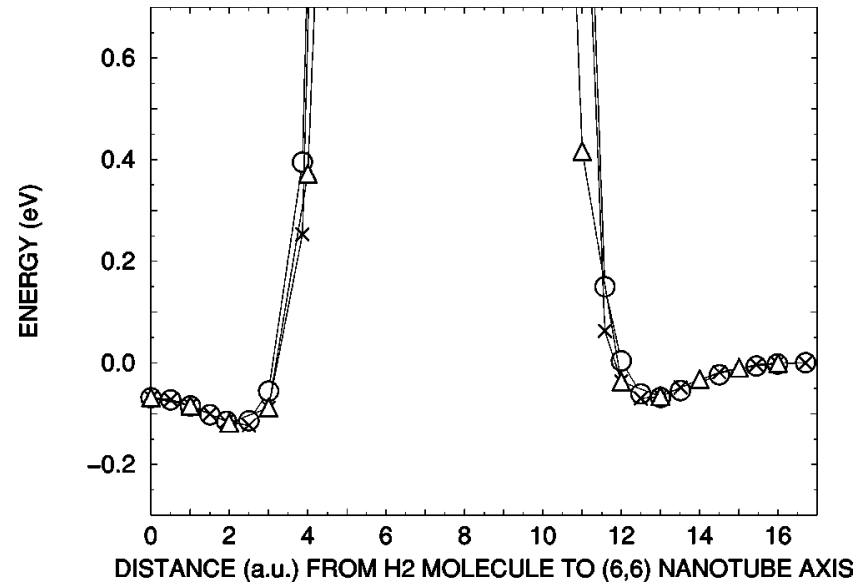

FIG. 6. Interaction energy of an $\mathrm{H}_{2}$ molecule and a $(6,6) \mathrm{SWCN}$, obtained in static calculations in which the $\mathrm{H}-\mathrm{H}$ and all the $\mathrm{C}-\mathrm{C}$ bond lengths are kept frozen, and only the distance $D_{a}$ between the molecule and the SWNT axis is varied. The approach of the molecule to the nanotube is upon the center of a hexagon of carbon atoms, with the axis of the molecule perpendicular (circles) and parallel (crosses) to the hexagon surface or upon a $\mathrm{C}-\mathrm{C}$ bond with the molecular axis parallel to that bond (triangles).

the fluorine atoms absorbed on the outer wall form broad continuous regions abruptly terminated in bands orthogonal to the tube axis. ${ }^{40}$ Very recent calculations by Bauschlicher ${ }^{22}$ for nanotubes covered by a high concentration of atomic hydrogen predict that the $\mathrm{H}$ atoms do not form random arrangements. Instead, some particular patterns are favored. For the $(10,0)$ nanotubes, a configuration of pairs of lines parallel to the nanotube axis, similar to our strip configuration, is favored over others like single lines, rings, or spirals. For $(5,5)$ nanotubes, pairs of lines are again more stable than ring configurations, although a zigzag pattern (related to the pair of lines configuration) is preferred.

Dynamical simulations have also been done for the hydrogen molecule inside the nanotube and the results are consistent with the physical picture provided by Fig. 3. The energy minimum occurs for the molecule physisorbed at the nanotube center. The $\mathrm{H}-\mathrm{H}$ bondlength is 1.48 a.u., and the binding energy of the molecule to the SWNT, measured with respect to the energy when the $\mathrm{H}_{2}$ molecule is far outside the nanotube, is $0.17 \mathrm{eV}$.

\section{INTERACTION OF $\mathrm{H}_{2}$ AND H WITH $(6,6)$ CARBON NANOTUBES: STATIC CALCULATIONS}

We have first studied the approach of a molecule to a $(6,6)$ carbon nanotube, with optimized radius $R=7.78$ a.u., keeping the interatomic distances frozen in both subsystems. The supercell contains 48 carbon atoms and has cell parameters $a=28.96$ a.u. and $c=9.28$ a.u., this one being twice as large as that used above for the $(5,5)$ nanotube. The energy cutoff was 40 Ry. We compare in Fig. 6 the potential energy curves when the center of mass of the molecule follows a radial path, perpendicular to the nanotube axis upon the center of a hexagon of carbon atoms. Two cases have been studied, with the molecular axis perpendicular (circles) and parallel (crosses) to the hexagon surface. The two curves are very close to each other and confirm the picture found for the

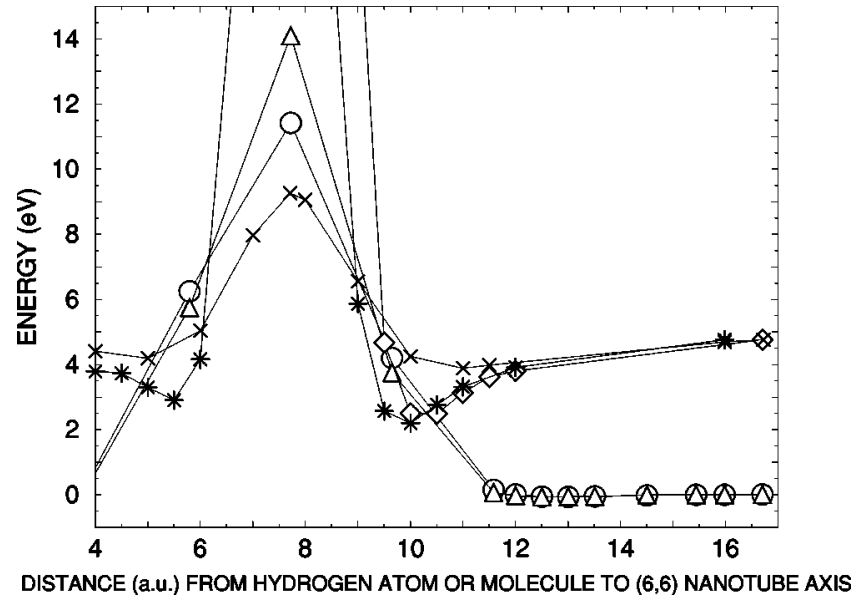

FIG. 7. Interaction energies for atomic and molecular hydrogen and a $(6,6)$ SWCN, obtained in static calculations similar to those reported in Fig. 6. The approach of the hydrogen atom is perpendicularly upon the midpoint of a $\mathrm{C}-\mathrm{C}$ bond (stars), upon the center of a hexagon (crosses), and upon a carbon atom (diamonds). The approach of the molecule is upon a hexagon with the molecular axis parallel (triangles) and perpendicular (circles). The energies of the curves for atomic $\mathrm{H}$ have been multiplied by a factor of 2 and shifted upwards (see text).

interaction with the $(5,5)$ nanotube: the molecule is weakly physisorbed both outside and inside the SWNT. The depths of the external and internal wells are $0.07 \mathrm{eV}$ and $0.12 \mathrm{eV}$, respectively. The binding energy in the external well is similar to that for the $(5,5) \mathrm{SWNT}$. A small difference is that the minimum for the molecule inside the nanotube does not occur at the position of the tube axis; it is displaced, instead, 2.5 a.u. from the axis. This is just a size effect arising from the larger radius, $R=7.78$ a.u., of the $(6,6)$ nanotube. For this reason, the depth of the inner minimum is, actually, smaller for this nanotube. To test the accuracy of these results, a calculation was performed with a cutoff of 70 Ry for the case when the molecule approaches the nanotube with the molecular axis perpendicular upon the center of a carbon hexagon. The minimum of the outer physisorption well occurs at $D=12.6$ a.u. and the binding energy is $0.07 \mathrm{eV}$, in agreement with the calculation with a cutoff of 40 Ry. Finally, in Fig. 6 we show another curve (triangles) for the parallel approach of the molecule upon a $\mathrm{C}-\mathrm{C}$ bond. That curve is very close to those for the molecule upon the center of the hexagon.

We have also calculated the energies for the system formed by a single hydrogen atom approaching radially towards the $(6,6)$ SWNT, and the results are given in Fig. 7. One path of approach is above a carbon atom (diamonds), a second one is perpendicular upon the midpoint of a $\mathrm{C}-\mathrm{C}$ bond (stars), and a third one upon the center of a hexagon (crosses). The corresponding potential energies have been multiplied by a factor of 2 , for the reasons already explained. The binding energies for $\mathrm{H}$ upon a $\mathrm{C}$ atom and upon a $\mathrm{C}-\mathrm{C}$ bond on the outer part of the nanotube are equal to 1.3 and $1.1 \mathrm{eV}$, respectively. The equilibrium $\mathrm{C}-\mathrm{H}$ bond distance in the first case is 2.5 a.u., nearly the same bond length found for the $(5,5)$ nanotube in the same configuration. The binding energy upon the center of a hexagon is only $0.4 \mathrm{eV}$. The barrier for the insertion of the $\mathrm{H}$ atom into the nanotube following a path through the center of a hexagon amounts to 
$2.3 \mathrm{eV}$. The barrier is evidently much larger if the $\mathrm{H}$ atom is forced to go through a $\mathrm{C}-\mathrm{C}$ bond, in agreement with the result for the $(5,5)$ SWNT (that barrier is about $15 \mathrm{eV}$; see Fig. 3). Ma et al. ${ }^{18,19}$ have estimated barriers for the insertion of $\mathrm{H}$ through the wall of $(5,5)$ nanotubes. They performed dynamical DFT simulations and also simulations based on phenomenological Tersoff potentials. The heights obtained are 16 and $14 \mathrm{eV}$ for the DFT and phenomenological insertion barriers, respectively. These are substantially higher than our calculated insertion barrier through the center of a hexagon of the $(6,6)$ SWNT. Part of the difference can be due to the fact that dynamical simulations only give an upper bound to the barrier. Notice again that all the H-SWNT potential energy curves appearing in Fig. 7 have been multiplied by a factor of 2 and shifted upwards by $4.76 \mathrm{eV}$, the dissociation energy of the $\mathrm{H}_{2}$ molecule. Those curves can then be interpreted as representing the interaction between two separated $\mathrm{H}$ atoms and the $(6,6) \mathrm{SWNT}$, and can be directly compared with the curves giving the interaction between the $\mathrm{H}_{2}$ molecule and the SWNT. Two of these have been plotted in Fig. 7 , corresponding to the molecule upon a hexagon. Physisorption of the $\mathrm{H}_{2}$ molecule is again predicted to be the ground state of the system, but the molecular and atomic curves cross at a distance $D_{w}=2.7$ a.u. from the nanotube wall. This means that when forced to approach the SWNT closer than this critical distance, the molecule dissociates. The dissociation barrier, estimated as the difference between the energy of the $\mathrm{H}_{2}$ molecule at the distance when the molecular and atomic curves cross and the energy of the molecule in its physisorption well, is $2.5 \mathrm{eV}$.

\section{INTERACTION OF $\mathrm{H}_{2}$ AND H WITH $(6,6)$ CARBON NANOTUBES: CALCULATIONS INCLUDING ATOMIC RELAXATION}

To investigate the effect of atomic relaxation we first allowed for the variation of the bond length of the molecule, keeping the atomic positions of the carbon atoms in the nanotube frozen. The supercell was the same one described in Sec. $\mathrm{V}$ above, that is, with $a=28.96$ a.u., $c=9.28$ a.u., and 48 carbon atoms. The molecule was placed above the center of a carbon hexagon, and for each distance $D_{a}$, the bond length $d$ was optimized. The configuration of minimum energy obtained in this way is essentially the same found in Sec. V, that is, with the molecule physisorbed at a distance $D_{a}$ equal to 12.6 a.u. and the bond length intact.

The calculations just reported were performed using a supercell containing 48 carbon atoms. Dynamical relaxations with this large simulation cell require an enormous amount of computing time, and to facilitate the calculations we have used smaller simulation cells with 24 carbon atoms ( $c$ $=4.64$ a.u.). We have performed dynamical simulation runs for the $\mathrm{H}_{2}$ molecule initially at different distances from the nanotube, in positions upon a $\mathrm{C}-\mathrm{C}$ bond. For initial configurations with the molecule at distances $D_{a}$ between 11 and 13 a.u., the simulations drive the molecule towards the minimum of the physisorption well. Both $D_{a}=13.2$ a.u. and the energy of the system are quite close to those obtained in the static calculations. Simulations for the molecule inside the nanotube also give results in agreement with the static cal-

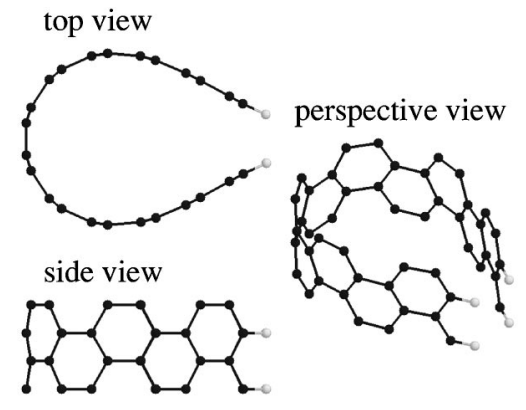

FIG. 8. Snapshot with the broken nanotube configuration for a dynamical simulation starting with the hydrogen molecule at a distance 1.2 a.u. from the external wall of a $(6,6)$ nanotube. Three views are provided. To improve the visualization of the system, the structure shown contains two simulation cells along the direction of the nanotube axis.

culations: dynamical runs with the molecule initially separated 4 a.u. from the nanotube axis lead to a final configuration with the molecule at 2.1 a.u. from that axis and a bond length of 1.48 a.u. A noticeable effect is that the transversal section of the tube becomes slightly deformed. Selected dynamical simulations were performed with a simulation cell containing 48 carbon atoms, because a larger cell implies a smaller interaction between the $\mathrm{H}_{2}$ molecules in different cells. The simulations with the molecule initially placed near the minimum of the outer physisorption well confirm the stability of the molecule in that region and show that the orientation of the molecular axis changes easily.

An interesting result is again obtained when the simulation begins with the hydrogen molecule dissociated ( $d$ $=4.1$ a.u.) outside the nanotube, at $D_{a}=9$ a.u. from the axis, that is, at a short distance of only $D_{w}=1.2$ a.u. from the nanotube wall. From the potential energy curves of Fig. 7 one can appreciate that this initial configuration corresponds to a state of high potential energy. Scission of the $\mathrm{C}-\mathrm{C}$ bond is again found, as for the $(5,5)$ nanotube, and a snapshot view is given in Fig. 8. To improve visualization of the system, the structure plotted includes two simulation cells. Each $\mathrm{H}$ atom ends up attached to a $\mathrm{C}$ atom, with $\mathrm{H}-\mathrm{C}$ bond lenghts of 2.07 a.u. Those two carbon atoms are originally nearest neighbors in the SWNT, and their separation in Fig. 8 is 5.76 a.u., strikingly larger than the bondlength 2.68 a.u. in the isolated nanotube. Similar results were obtained for other initial configurations of the $\mathrm{H}_{2}$ molecule close to the nanotube wall.

We expect a similar breaking of $\mathrm{C}-\mathrm{C}$ bonds if a larger supercell in the direction parallel to the nanotube axis were used, but in those cases the nanotube wall will be shattered only locally because of the lower hydrogen concentration. Those localized scissures, or the grouping of several broken bond defects to produce a larger opening, could provide a way of allowing foreign species to enter the nanotube through a shattered wall. Also the weakening of $\mathrm{C}-\mathrm{C}$ bonds could provide a hint to explain the mechanism for the doubling of SWNT diameters observed by Nikolaev et al. ${ }^{41}$ in nanotube ropes. In those experiments, SWNT ropes were annealed at temperatures up to $1773 \mathrm{~K}$ in a hydrogen atmosphere, and it was observed that some neighbor nanotubes coalesce, leading to the formation of tubes of larger diam- 
eters. According to our results, hydrogen could catalyze the coalescence of adjacent nanotubes. In that process, the weakening of $\mathrm{C}-\mathrm{C}$ bonds by hydrogen attack and the selforganization of the weakened bonds along a line parallel to the nanotube axis could provide the first step. A driving mechanism for the self-organization of those defects along a stripe would be the establishment of bonding links between adjacent nanotubes, mediated perhaps by the hydrogen atoms. The cooperative effect of those self-organized bonding patches might lead to the coalescence of adjacent nanotubes, with the corresponding increase of the diameter. However, intensive work remains to be done to investigate those processes.

\section{CONCLUSIONS}

The static calculations and dynamical simulations of the interaction of molecular and atomic hydrogen with $(5,5)$ and $(6,6)$ nanotubes provide the following picture: the lowestenergy states of those systems are achieved for the molecules weakly physisorbed either outside or inside the nanotube. This is the main result of our work since molecular physisorption is relevant for the purposes of hydrogen storage in porous graphitic materials. When the molecule has enough kinetic energy to approach closely to the nanotube wall, then the molecule dissociates and the two hydrogen atoms bind to two nearest-neighbor carbon atoms. This dissociative chemisorption weakens that particular $\mathrm{C}-\mathrm{C}$ bond. The weakening of the $\mathrm{C}-\mathrm{C}$ bond, combined with an excess kinetic energy of the incoming molecule, can actually break the $\mathrm{C}-\mathrm{C}$ bond, shattering locally the nanotube. Self-organization of a large concentration of those defects can lead to the scission of the nanotube along a line parallel to the nanotube axis.

\section{ACKNOWLEDGMENTS}

This work was supported by DGESIC (Grant No. PB980345) and European Community (RTN-COMELCAN). We acknowledge the computational facilities provided by CESCA and CEPBA. We are grateful to Professor R. Pedrosa and Professor P. Espinet for enlightening discussions.

${ }^{1}$ A. C. Dillon, K. M. Jones, T. A. Bekkedahl, C. H. Kiang, D. S. Bethune, and M. J. Heben, Nature (London) 386, 377 (1997).

${ }^{2}$ S. Hynek, W. Fuller, and J. Bentley, Int. J. Hydrogen Energy 22, 601 (1997).

${ }^{3}$ F. Darkrim and D. Levesque, J. Chem. Phys. 109, 4981 (1998); F. Darkrim, J. Vermesse, P. Malbrunot, and D. Levesque, ibid. 110, 4020 (1999).

${ }^{4}$ Y. Ye, C. C. Ahn, C. Witham, B. Fultz, J. Liu, A. G. Rinzler, D. Colbert, K. A. Smith, and R. E. Smalley, Appl. Phys. Lett. 74, 2307 (1999).

${ }^{5}$ C. Liu, Y. Y. Fan, M. Liu, H. T. Cong, H. M. Cheng, and M. S. Dresselhaus, Science 286, 1127 (1999).

${ }^{6}$ A. Chambers, C. Park, R. T. K. Baker, and N. M. Rodriguez, J. Phys. Chem. B 102, 4523 (1998).
${ }^{7}$ Q. Wang and J. K. Johnson, J. Chem. Phys. 110, 577 (1999); J. Phys. Chem. B 103, 4809 (1999).

${ }^{8}$ P. Chen, X. Wu, J. Lin, and K. L. Tan, Science 285, 91 (1999).

${ }^{9}$ R. T. Yang, Carbon 38, 623 (2000).

${ }^{10}$ A. Cao, H. Zhu, X. Zhang, X. Li, D. Ruan, C. Xu, B. Wei, J. Liang, and D. Wu, Chem. Phys. Lett. 342, 510 (2001).

${ }^{11}$ U. Bünger and W. Zittel, Appl. Phys. A: Mater. Sci. Process. 72, 147 (2001).

${ }^{12}$ M. Hirscher, M. Becher, M. Haluska et al., Appl. Phys. A: Mater. Sci. Process. 72, 129 (2001)

${ }^{13}$ A. C. Dillon and M. J. Heben, Appl. Phys. A: Mater. Sci. Process. 72, 133 (2001).

${ }^{14}$ B. N. Khare, M. Meyyappan, A. M. Cassell, C. V. Nguyen, and J. Han, Nano Lett. 2, 73 (2002).

${ }^{15}$ V. Meregalli and M. Parrinello, Appl. Phys. A: Mater. Sci. Process. 72, 143 (2001).

${ }^{16}$ V. V. Simonyan, P. Diep, and J. K. Johnson, J. Chem. Phys. 111, 9778 (1999).

${ }^{17}$ G. Stan and M. W. Cole, J. Low Temp. Phys. 110, 539 (1998).

${ }^{18}$ Y. Ma, Y. Xia, M. Zhao, R. Wang, and L. Mei, Phys. Rev. B 63, 115422 (2001).

${ }^{19}$ Y. Ma, Y. Xia, M. Zhao, M. Ying, X. Liu, and P. Liu, J. Chem. Phys. 115, $8152(2001)$

${ }^{20}$ L. Jeloaica and V. Sidis, Chem. Phys. Lett. 300, 157 (1999).

${ }^{21}$ C. W. Bauschlicher, Jr., Chem. Phys. Lett. 322, 237 (2000).

${ }^{22}$ C. W. Bauschlicher, Nano Lett. 1, 223 (2001); C. W. Bauschlicher and C. So, Nano Lett. 2, 337 (2002).

${ }^{23}$ S. M. Lee and Y. H. Lee, Appl. Phys. Lett. 76, 2877 (2000).

${ }^{24}$ O. Gülseren, T. Yildirim, and S. Ciraci, Phys. Rev. Lett. 87, 116802 (2001); T. Yildirim, O. Gülseren, and S. Ciraci, Phys. Rev. B 64, 075404 (2001).

${ }^{25}$ J. S. Arellano, L. M. Molina, A. Rubio, and J. A. Alonso, J. Chem. Phys. 112, 8114 (2000).

${ }^{26}$ K. Tada, S. Furuya, and K. Watanabe, Phys. Rev. B 63, 155405 (2001).

${ }^{27}$ P. Dubot and P. Cenedese, Phys. Rev. B 63, 241402 (2001).

${ }^{28}$ S. P. Chan, G. Chen, X. G. Gong, and Z. F. Liu, Phys. Rev. Lett. 87, 205502 (2001).

${ }^{29}$ Y. Okamoto and Y. Miyamoto, J. Phys. Chem. B 105, 3470 (2001).

${ }^{30}$ M. B. Nardelli, Solid State Commun. 97, 215 (1996).

${ }^{31}$ M. Bockstedte, A. Kley, J. Neugebauer, and M. Scheffler, Comput. Phys. Commun. 107, 187 (1997).

${ }^{32}$ J. P. Perdew and A. Zunger, Phys. Rev. B 23, 5048 (1981).

${ }^{33}$ G. B. Bachelet, D. R. Hamann, and M. Schlüter, Phys. Rev. B 26, 4199 (1982); D. R. Hamann, ibid. 40, 2980 (1989).

${ }^{34}$ C. M. Brown, T. Yildirim, D. A. Newmann, M. J. Heben, T. Gennett, A. C. Dillon, J. L. Alleman, and J. E. Fischer, Chem. Phys. Lett. 329, 311 (2000).

${ }^{35}$ N. D. Lang, Phys. Rev. Lett. 46, 842 (1981).

${ }^{36}$ M. C. Schabel and J. L. Martins, Phys. Rev. B 46, 7185 (1992).

${ }^{37}$ J. C. Charlier, X. Gonze, and J. P. Michenaud, Europhys. Lett. 29, 43 (1995).

${ }^{38}$ J. M. Pacheco and J. P. Prates Ramalho, Phys. Rev. Lett. 79, 3873 (1997).

${ }^{39}$ X. Fan, E. C. Dickey, P. C. Eklund, K. A. Williams, L. Grigorian, R. Buczko, S. T. Pantelides, and S. J. Pennycook, Phys. Rev. Lett. 84, 4621 (2000).

${ }^{40}$ K. F. Kelly, I. W. Chiang, E. T. Mickelson, R. H. Hauge, J. L. Margrave, X. Wang, G. E. Scuseria, C. Radloff, and N. J. Halas, Chem. Phys. Lett. 313, 445 (1999).

${ }^{41}$ P. Nikolaev, A. Thess, A. G. Rinzler, D. T. Colbert, and R. E. Smalley, Chem. Phys. Lett. 266, 422 (1997). 